\title{
Accurate BER Analysis of Asynchronous DS-CDMA Systems in Ricean Channels
}

\author{
Xiang Liu and Lajos Hanzo \\ School of Electronics and Computer Science, University of Southampton, SO17 1BJ, UK \\ lh@ecs.soton.ac.uk, www-mobile.ecs.soton.ac.uk
}

\begin{abstract}
The accurate Bit Error Rate (BER) calculation of an asynchronous Ricean-faded DS-CDMA system using random spreading sequences and BPSK modulation is studied in this paper. A new closed-form expression is derived for the conditional characteristic function of the cochannel interference. Furthermore, a new BER expression involving only a single integration is derived invoking the characteristic function approach and hypergeometric functions of two variables. The accuracy of our BER expression is confirmed by our simulation results for various spreading sequence lengths and various Ricean $K$-factors. By contrast, the Standard Gaussian Approximation (SGA) overestimates the BER of Rayleigh fading channels, when there is no Line-of-Sight (LOS) component and it under-estimates the BER, when the LOS component becomes stronger.
\end{abstract}

\section{INTRODUCTION}

The Bit Error Ratio (BER) is one of the most important performance metric in communication systems and hence it has been extensively studied in various contexts.

In Direct Sequence Code Division Multiple Access (DSCDMA) systems [1], various versions of the so-called Gaussian approximation are widely used for modeling the distribution of the Multiple Access Interference (MAI). A few examples are the Standard Gaussian Approximation (SGA) [2][15], the Improved Gaussian Approximation (IGA) [2], [6], [9], [10], [12], [13], [15]-[17], the Simplified IGA (SIGA) [2], [6], [9], [11], [13], [15], and the Improved Holtzman Gaussian Approximation (IHGA) [11]. However, the accuracy of the various Gaussian approximation techniques depends on the specific configuration of the system. It is well known that the Gaussian approximation techniques become less accurate, when a low number of users is supported or when there is a dominant interferer [12].

Therefore the accurate BER analysis dispensing with the previous assumptions on the MAI distribution is desirable. Hence a number of accurate techniques have been developed, such as the series expansion [12], [13], [18]-[20], the Fourier [2], [3], [11], [15], [18] and Laplace [21] transform based methods. The latter two lead to the Characteristic Function (CF) and Moment Generating Function (MGF) based approaches and have been prevalent in the accurate BER analysis of communication systems.

The BER performance of DS-CDMA systems communicating over Additive White Gaussian Noise (AWGN) channels has been extensively studied [5]-[10], [12], [13], [15], [16],

The financial support of the EPSRC, UK and the EU in the framework of the NEWCOM, NEXWAY and PHOENIX projects is gratefully acknowledged.
[18]-[20], [22]-[26], and there are numerous studies also for transmission over both Rayleigh [2], [12] and Nakagami$m$ [3], [4], [11], [17], [21] channels. Geraniotis and Pursley [18] were the first authors, who investigated the accurate BER calculation of asynchronous DS-CDMA systems over AWGN channels using the CF approach. Then Cheng and Beaulieu extended the results to both Rayleigh [2] and Nakagami- $m$ [3] channels.

However, to the authors' best knowledge, the accurate BER analysis of asynchronous Ricean-faded DS-CDMA systems using random spreading [2]-[17], [19], [20] sequences is still an open problem. There are many propagation environments, such as microcellular urban and suburban land mobile, as well as picocellular indoor and factory scenarios, where there exist Line-Of-Sight (LOS) propagation paths between the transmitter and the receiver [27]. In the presence of a LOS component, the Ricean distribution, also known as the Nakagami- $n$ distribution [28], [29], is a better model of the fading channel. The Ricean distribution becomes the Rayleigh distribution, when the energy of the LOS component becomes zero [27], [30]. The novel contribution of this paper is that we provide an accurate BER expression for asynchronous DS-CDMA systems in a Ricean fading environment, which requires only a single numerical integration, when using the hypergeometric functions of two variables [31]-[33].

The organization of this paper is as follows. In Section II an asynchronous DS-CDMA system using BPSK modulation is considered in the context of a Ricean fading channel. Then in Section III an accurate BER expression based on the characteristic function approach is derived for the BER calculation of the system using random spreading sequences. In Section IV our numerical results are presented and finally, in Section V our conclusions are provided.

\section{System And Channel Model}

We consider an asynchronous Ricean-faded DS-CDMA system using BPSK modulation, random spreading sequences and rectangular chip waveforms. The rectangular pulse having a duration of $T$ is defined as:

$$
p_{T}(t)=\left\{\begin{array}{l}
1, t \in[0, T) \\
0, \text { otherwise }
\end{array}\right.
$$

Each of the $K$ users is assigned a binary random spreading sequence $\left\{a_{k, m}\right\}_{m=-\infty}^{\infty}$, where $\left\{a_{k, m}\right\}_{m=0}^{L-1}$ are mutually independent and symmetrically Bernoulli distributed [34], implying that we have $P\left\{a_{k, m}= \pm 1\right\}=\frac{1}{2}$, and $L$ is the number 
of chips in the random spreading sequences. The $k$ th user's binary data sequence of $\left\{b_{k, m}\right\}_{m=-\infty}^{\infty}$, which is also mutually independent and symmetrically Bernoulli distributed, BPSK modulates the phase of the the carrier. The complex lowpass equivalent of the received signal, $\widetilde{r}(t)$, may be written as:

$\widetilde{r}(t)=\sum_{k=0}^{K} \sqrt{2 P_{t}} a_{k}\left(t-\tau_{k}\right) b_{k}\left(t-\tau_{k}\right) \widetilde{h}_{k} e^{j\left[\omega_{c}\left(t-\tau_{k}\right)+\theta_{k}\right]}+\widetilde{\eta}(t)$

where $P_{t}$ and $\omega_{c}$ are the average transmitted power and the carrier's angular frequency, respectively, both of which are common to all users. Furthermore, $a_{k}(t)=\sum_{m=-\infty}^{\infty} a_{k, m} p_{T_{c}}(t-$ $\left.m T_{c}\right)$ and $b_{k}(t)=\sum_{m=-\infty}^{\infty} b_{k, m} p_{T_{s}}\left(t-m T_{s}\right)$ represents the $k$ th user's spreading signal and data signal, respectively, $T_{c}$ and $T_{s}$ are the chip duration and bit duration, respectively, satisfying $T_{s}=L T_{c},\left\{\theta_{k}\right\}_{k=0}^{K-1}$ and $\left\{\tau_{k}\right\}_{k=0}^{K-1}$ are the carrier phase shift and the time delay, respectively, which are independently and uniformly distributed in $[0,2 \pi)$ and $\left[0, T_{s}\right)$, respectively. Finally, $\widetilde{\eta}(t)$ is the zero-mean stationary complex-valued Additive White Gaussian Noise (AWGN) having a double-sided power spectral density of $N_{0}$.

The received signal of each user suffers from independent Ricean fading, which is characterized by the complex-valued random variable $\widetilde{h}_{k}=h_{k} e^{j \varphi_{k}}$. The Ricean fading process physically consists of a direct LOS component having a power of $\mu_{k}^{2}$ and many weaker components having a total power of $2 \sigma_{k}^{2}$. Hence the Probability Density Function (PDF) of its modulus, $h_{k}$, is given by [28], [29]:

$$
f\left(h_{k}\right)=\frac{h_{k}}{\sigma_{k}^{2}} \exp \left(-\frac{h_{k}^{2}+\mu_{k}^{2}}{2 \sigma_{k}^{2}}\right) \mathbb{I}_{0}\left(\frac{h_{k} \mu_{k}}{\sigma_{k}^{2}}\right), h_{k} \geq 0,
$$

where we have $\mu_{k} \geq 0$ and $\sigma_{k}>0$, and $\mathbb{I}_{0}(x)$ is the zerothorder modified Bessel function of the first kind [35]. Another alternative expression is also often used [27], [30]. The Ricean $K$-factor is defined as:

$$
\kappa_{k}=n_{k}^{2}=\frac{\mu_{k}^{2}}{2 \sigma_{k}^{2}} .
$$

where $n_{k}$ is the Ricean fading parameter. Using Horn's confluent hypergeometric function of two variables [31], [33], we may derive the characteristic function of the modulus $h_{k}$ as:

$$
\begin{aligned}
& \Phi_{h_{k}}(\omega)=\exp \left(-\frac{\mu_{k}^{2}}{2 \sigma_{k}^{2}}\right) \Psi_{2}\left(1 ; 1, \frac{1}{2} ; \frac{\mu_{k}^{2}}{2 \sigma_{k}^{2}},-\frac{1}{2} \sigma_{k}^{2} \omega^{2}\right) \\
& +j \sqrt{2} \sigma_{k} \omega \exp \left(-\frac{\mu_{k}^{2}}{2 \sigma_{k}^{2}}\right) \Psi_{2}\left(\frac{3}{2} ; 1, \frac{3}{2} ; \frac{\mu_{k}^{2}}{2 \sigma_{k}^{2}},-\frac{1}{2} \sigma_{k}^{2} \omega^{2}\right),
\end{aligned}
$$

where $\Psi_{2}\left(\alpha ; \gamma, \gamma^{\prime} ; x, y\right)$ is one of Horn's confluent hypergeometric functions of two variables [31], [33]. Equation 5 is a new closed-form expression, which is equivalent to the expression of a sum of infinite series provided by Table II in [30].

The phase $\varphi_{k}$ is neither uniform distributed nor independent of the modulus $h_{k_{\sim}}$ [29]. However the complex-valued Ricean random variable $\widetilde{h}_{k}$ may be decomposed into the sum of two independent real-valued Gaussian variables according to $\widetilde{h}_{k}=h_{k x}+j h_{k y}$. The mean and variance of $h_{k x}$ and $h_{k y}$ are $\left\{\mu_{k x}, \sigma_{k}^{2}\right\}$ and $\left\{\mu_{k y}, \sigma_{k}^{2}\right\}$, respectively, where we have $\mu_{k x}^{2}+\mu_{k y}^{2}=\mu_{k}^{2}$.

\section{ACCURATE BER ANALYSIS}

\section{A. Receiver Statistic}

In the case of coherent demodulation as well as perfect phase and chip synchronization, the decision statistic derived for the 0th user's signal is given by:

$$
Z=h_{0} L b_{0,0}+\sum_{k=1}^{K-1} \Re\left\{X_{k} \widetilde{h}_{k} e^{j \Delta_{k}}\right\}+\eta,
$$

where $\Re\{\widetilde{x}\}$ denotes the real part of the complex number $\widetilde{x}$, while $\Delta_{k}=-\omega_{c}\left(\tau_{k}-\tau_{0}\right)+\left(\theta_{k}-\theta_{0}\right)$ denotes the phase shift difference between the $k$ th and 0th user, which is uniformly distributed in $[0,2 \pi)$. The noise component $\eta$ can be shown to be a zero-mean Gaussian distributed random variable having a variance of $\sigma_{\eta}^{2}=\frac{N_{0} L}{T_{c}}$. The random variable $X_{k}$ is defined as [2], [7]:

$$
\begin{aligned}
X_{k}= & \sum_{m=0}^{L-2} Y_{k, m}\left[\left(1-\nu_{k}\right)+a_{0, m} a_{0, m+1} \nu_{k}\right] \\
& +Y_{k, L-1} \nu_{k}+Y_{k, L}\left(1-\nu_{k}\right),
\end{aligned}
$$

where the $(L+1)$ random variables $\left\{Y_{k, m}\right\}_{m=0}^{L}$ are mutually independent and symmetric Bernoulli distributed, conditioned on the 0th user's spreading sequence $\left\{a_{0, m}\right\}_{m=0}^{L-1}$. Furthermore, the relative chip shifts between the $k$ th and 0th user normalized by the chip duration, $\nu_{k}$, are mutually independent and uniformly distributed in $[0,1)$. The $(L-1)$ possible chip transitions of $\left\{a_{0, m} a_{0, m+1}\right\}_{m=0}^{L-2}$ can be categorized into two sets according to whether there is a chip value change or not [2], [7]. Let $B$ and $A$ denote the number of chip boundaries both with and without chip-value transitions within the 0th user's spreading sequence, respectively. Then we have $A+B=L-1$.

\section{B. BER Calculation}

The Co-Channel Interference (CCI) $I_{k}=\Re\left\{X_{k} \widetilde{h}_{k} e^{j \Delta_{k}}\right\}$ imposed by the different interferers is mutually independent, conditioned on $B$ [2], [7]. Upon defining the total interference plus noise as $\xi=\sum_{k=1}^{K-1} I_{k}+\eta[2]$, it transpires that both its PDF $f_{\xi \mid B}(x)$ and its $\operatorname{CF} \Phi_{\xi \mid B}(\omega)=\Phi_{\eta}(\omega) \prod_{k=1}^{K-1} \Phi_{I_{k} \mid B}(\omega)$ are even. Hence the Cumulative Distribution Function (CDF) $\mathrm{F}_{\xi \mid B}(x)$ of the CCI can be shown to be:

$$
\mathrm{F}_{\xi \mid B}(x)=\frac{1}{2}+\frac{1}{2 \pi} \int_{-\infty}^{\infty} \frac{\sin (\omega x)}{\omega} \Phi_{\xi \mid B}(\omega) \mathrm{d} \omega .
$$

Furthermore, the 0th user's Bit Error Probability (BEP) $P_{e \mid B}$ conditioned on $B$ may be shown to be:

$$
P_{e \mid B}=\int_{-\infty}^{\infty}\left[1-\mathrm{F}_{\xi \mid B}(x L)\right] f_{h_{0}}(x) \mathrm{d} x,
$$


where $f_{h_{0}}(x)$ is the PDF of the 0th user's fading amplitude, $h_{0}$, given by Equation 3. Applying the definition of the characteristic function [34] and the Fourier transform pair 8 in Table 17.23 of [35] as well as exchanging the order of the integrals, Equation 9 may be simplified to:

$$
P_{e \mid B}=\frac{1}{2}-\frac{1}{\pi} \int_{0}^{\infty} \frac{1}{\omega} \Phi_{\xi \mid B}(\omega) \Im\left\{\Phi_{h_{0}}(\omega L)\right\} \mathrm{d} \omega,
$$

where $\Im\left\{\Phi_{h_{0}}(\omega)\right\}$ is the imaginary part of the CF of the 0th user's fading amplitude, $h_{0}$, which was given by Equation 5 . Alternatively, Equation 10 may also be derived by applying Parseval's theorem similar to the approaches of [30], [36]. Note that Equation 10 applies not only to Ricean fading, but to any arbitrarily distributed fading, whenever $f_{\xi \mid B}(x)$, or equivalently $\Phi_{\xi \mid B}(\omega)$, is even. For example, when Rayleigh or Nakagami- $m$ fading is considered, Equation 10 reduces to Equation 39 of [2] and to Equation 21 of [3], respectively.

Finally, the overall average BEP is obtained by averaging $P_{e \mid B}$ over all spreading sequences, yielding:

$$
P_{e}=2^{-(L-1)} \sum_{B=0}^{L-1}\left(\begin{array}{c}
L-1 \\
B
\end{array}\right) P_{e \mid B}
$$

\section{MAI Analysis}

The only task that remained unsolved so far is the determination of the conditional $\mathrm{CF}$ of the co-channel interference incurred by the $k$ th user, $\Phi_{I_{k} \mid B}(\omega)$. As seen in Equation 10, only the characteristic function range spanning over $\omega \geq$ 0 is considered, which will be justified during our further discourse.

Upon exploiting the fact that Ricean random variables are composed of two independent Gaussian random variables, as described in Section II, it can be readily shown that the characteristic function of $I_{k}$ conditioned on $X_{k}$ and $\Delta_{k}$ may be expressed as:

$\Phi_{I_{k} \mid X_{k}, \Delta_{k}}(\omega)=\exp \left[j X_{k} \mu_{k} \omega \cos \left(\Delta_{k}+\vartheta_{k}\right)-\frac{1}{2} X_{k}^{2} \sigma_{k}^{2} \omega^{2}\right]$

where $\vartheta_{k}$ satisfies $\cos \vartheta_{k}=\frac{\mu_{k x}}{\mu_{k}}$ and $\sin \vartheta_{k}=\frac{\mu_{k y}}{\mu_{k}}$. Averaging $\Phi_{I_{k} \mid X_{k}, \Delta_{k}}(\omega)$ over $\Delta_{k} \in[0,2 \pi)$ with the aid of Equation 3.339 in [35], we have the CF of $I_{k}$ conditioned on $X_{k}$ in the following form:

$$
\Phi_{I_{k} \mid X_{k}}(\omega)=\exp \left(-\frac{1}{2} X_{k}^{2} \sigma_{k}^{2} \omega^{2}\right) \mathbb{J}_{0}\left(X_{k} \mu_{k} \omega\right),
$$

where $\mathbb{J}_{0}(x)$ is the zeroth-order Bessel function of the first kind [35], which has a relation with $\mathbb{I}_{0}(x)$ as $\mathbb{I}_{0}(x)=\mathbb{J}_{0}(j x)$. Finally, the conditional CF, $\Phi_{I_{k} \mid B}(\omega)$, is obtained by averaging $\Phi_{I_{k} \mid X_{k}}(\omega)$ over $\left\{Y_{k, m}\right\}_{m=0}^{L}$ and $\nu_{k}$ as follows:

$$
\begin{gathered}
\Phi_{I_{k} \mid B}(\omega)=2^{-(L+1)} \sum_{d_{1} \in \mathcal{A}} \sum_{d_{2} \in \mathcal{B}}\left(\begin{array}{c}
A \\
\frac{d_{1}+A}{2}
\end{array}\right)\left(\begin{array}{c}
B \\
\frac{d_{2}+B}{2}
\end{array}\right) \\
\times \sum_{Y_{k, L-1}, Y_{k, L} \in\{ \pm 1\}} \Phi_{I_{k} \mid \lambda_{0}, \lambda_{1}}(\omega),
\end{gathered}
$$

where the two sets $\mathcal{A}, \mathcal{B}$ and the coefficients $\lambda_{0}, \lambda_{1}$ are defined as [2], [7]:

$$
\begin{aligned}
& \mathcal{A}=\{-A,-(A-2), \ldots, A-2, A\}, \\
& \mathcal{B}=\{-B,-(B-2), \ldots, B-2, B\}, \\
& \lambda_{0}=d_{1}+d_{2}+Y_{k, L} \\
& \lambda_{1}=-2 d_{2}+Y_{k, L-1}-Y_{k, L} .
\end{aligned}
$$

The conditional CF, $\Phi_{I_{k} \mid \lambda_{0}, \lambda_{1}}(\omega)$, may be shown to be:

$$
\begin{aligned}
& \Phi_{I_{k} \mid \lambda_{0}, \lambda_{1}}(\omega)= \\
& \begin{cases}\frac{x}{\lambda_{1}} \mathbb{F}_{1: 0 ; 0}^{1: 0 ; 1}\left(\left.\frac{\frac{1}{2}:-;-;}{\left.\frac{3}{2}:-; 1 ;-\frac{1}{2} \sigma_{k}^{2} \omega^{2} x^{2},-\frac{1}{4} \mu_{k}^{2} \omega^{2} x^{2}\right)}\right|_{\lambda_{0}} ^{\lambda_{0}+\lambda_{1}}\right. \\
\operatorname{lr} \neq 0, \\
\lambda_{1} \neq 0 \\
\lambda_{1}=0,\end{cases}
\end{aligned}
$$

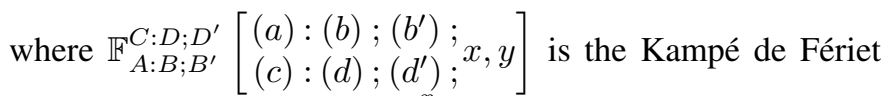
function [32], [33] and $\left.f(x)\right|_{x_{1}} ^{x_{2}}=f\left(x_{2}\right)-f\left(x_{1}\right)$. When $\mu_{k}=$ 0 or equivalently $\kappa_{k}=0$, i.e. when we experience Rayleigh fading, Equation 16 reduces to the results of [2].

\section{NumericAl RESUlts}

In this section we will compare our simulation results to those obtained from our accurate BER analysis in Section III as well as to those generated by the SGA approach.

Figures 1 and 2 illustrate the average BER performance versus the number of users, when the effects of background noise are ignored. Figure 1 compares the results obtained from our accurate BER analysis to our simulation results and shows that they match very well both for various spreading sequence lengths and for various Ricean $K$-factors. On the other hand, Figure 2 compares the results obtained using the SGA to our simulation results and shows an interesting phenomenon. It is widely recognized that the SGA slightly over-estimates the average BER, when the Ricean $K$-factor is $\kappa=0$, i.e. for Rayleigh fading. This has also been reported in [2]. By contrast, when $\kappa$ increases to 9 , the SGA under-estimates the average BER. Although not shown explicitly here, if we have $\kappa \rightarrow \infty$, which corresponds to having no fading and no noise, only CCI, the SGA will more severely under-estimate the average BER, which has been reported in the context of AWGN channels [12].

Figures 3 and 4 illustrate the average BER performance versus the per-bit SNR, when the number of users is $K=4$. Figure 3 compares the results obtained from our accurate BER analysis to the simulation results and shows that they match very well for both different spreading sequence lengths and for various Ricean fading parameters. On the other hand, Figure 4 compares the results obtained by the SGA to our simulation results and shows similar performance trends to those seen in Figure 2, when the Ricean $K$-factor $\kappa$ increases. Furthermore, the SGA still fails to accurately evaluate the average BER performance, particularly when the SNR is high.

\section{CONClusion}

We studied the accurate BER calculation of an asynchronous DS-CDMA system exposed to Ricean fading using random 


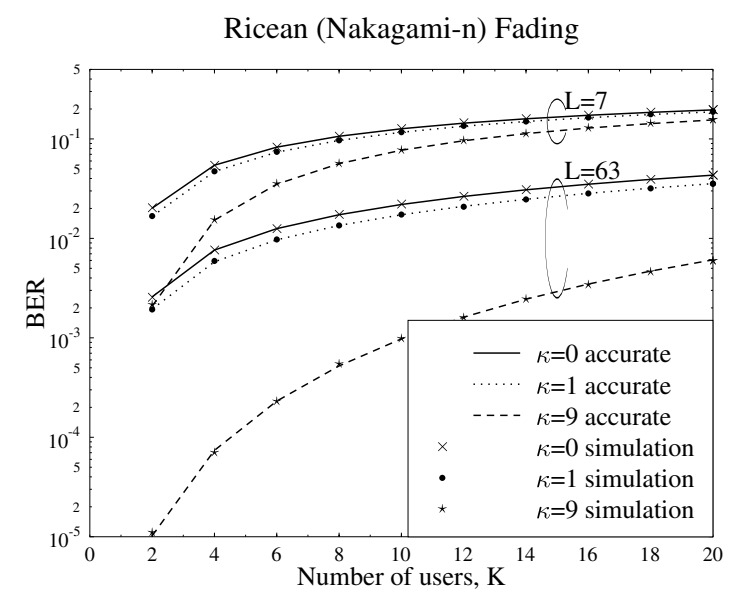

Fig. 1. BER versus the number of users $K$ in an asynchronous DS-CDMA system exposed to Ricean fading using random spreading sequences and BPSK modulation. The length of the random spreading sequences is $L=7$ and 63 . The Ricean $K$-factor is $\kappa=0,1$ and 9 , which is common to all users. The average power of all users at the receiver is equal and the background noise is ignored, i.e. we have $\gamma_{\mathrm{SNR}}=\infty$.

Ricean (Nakagami-n) Fading

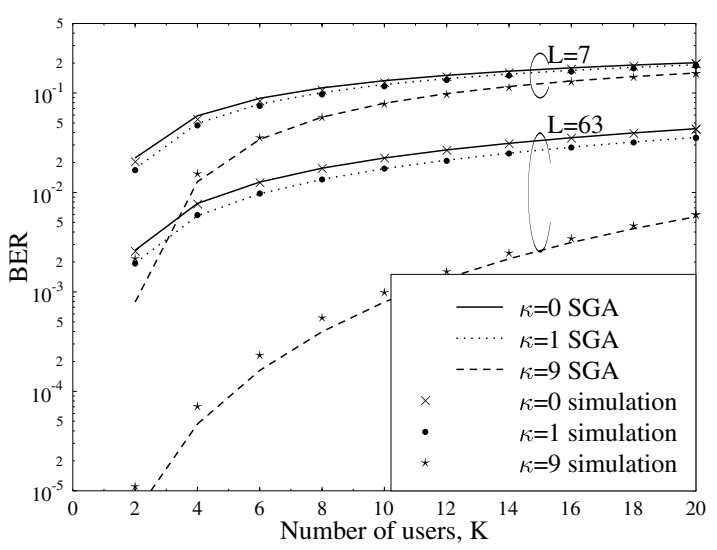

Fig. 2. BER versus the number of users $K$ in an asynchronous DS-CDMA system exposed to Ricean fading using random spreading sequences and BPSK modulation. The length of the random spreading sequences is $L=7$ and 63 . The Ricean $K$-factor is $\kappa=0,1$ and 9, which is common to all users. The average power of all users at the receiver is equal and the background noise is ignored, i.e. we have $\gamma_{\mathrm{SNR}}=\infty$.

spreading sequences and BPSK modulation. Using the CF approach and hypergeometric functions of two variables [31][33], we derived a new closed-form expression for the conditional $\mathrm{CF}$ of the cochannel interference and a single integration for the BER calculation. The accuracy of our BER expression was confirmed by our simulation results both for various spreading sequence lengths and for various Ricean $K$-factors. By contrast, the SGA approach over-estimates the BER, when the Ricean $K$-factor is $\kappa=0$ for Rayleigh fading, while it under-estimates the BER, when $\kappa$ increases. The inaccuracy of the SGA was also demonstrated, which becomes more
Ricean (Nakagami-n) Fading

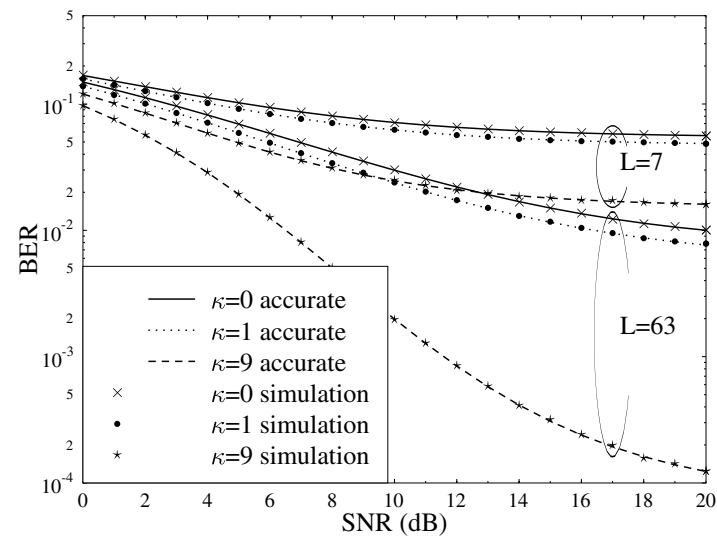

Fig. 3. BER versus per-bit SNR in an asynchronous DS-CDMA system exposed to Ricean fading using random spreading sequences and BPSK modulation. The length of the random spreading sequences is $L=7$ and 63 . The Ricean $K$-factor is $\kappa=0,1$ and 9 , which is common to all users. The average power of all users at the receiver is equal. The number of users is $K=4$.

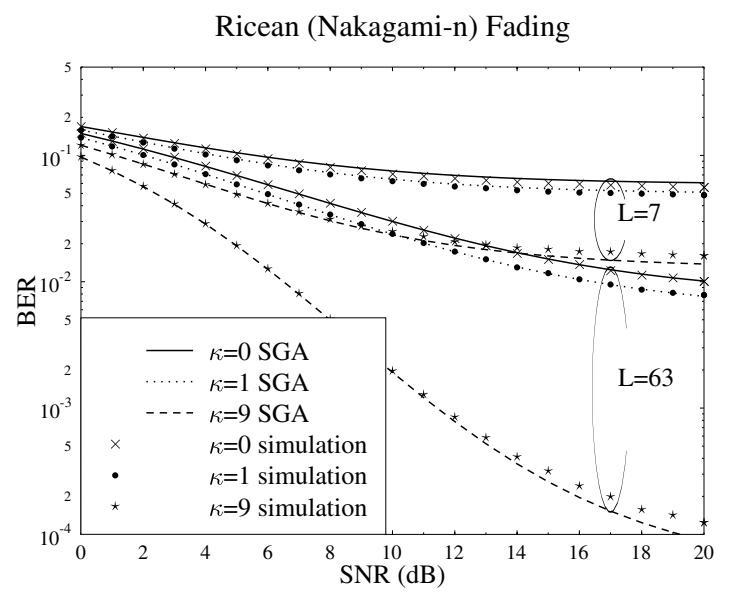

Fig. 4. BER versus per-bit SNR in an asynchronous DS-CDMA system exposed to Ricean fading using random spreading sequences and BPSK modulation. The length of the random spreading sequences is $L=7$ and 63. The Ricean $K$-factor is $\kappa=0,1$ and 9, which is common to all users. The average power of all users at the receiver is equal. The number of users is $K=4$.

prevalent when a low number of interferers is encountered and short spreading sequences are used.

\section{REFERENCES}

[1] L. Hanzo, L.-L. Yang, E.-L. Kuan, and K. Yen, Single and Multi-Carrier DS-CDMA : Multi-User Detection, Space-Time Spreading, Synchronisation, Networking and Standards. John Wiley and Sons Ltd., 2003.

[2] J. Cheng and N. Beaulieu, "Accurate DS-CDMA Bit-Error Probability Calculation in Rayleigh Fading," IEEE Transactions on Wireless Communications, vol. 1, no. 1, pp. 3-15, January 2002.

[3] — "Precise Bit Error Rate Calculation for Asynchronous DS-CDMA in Nakagami Fading," in IEEE Global Telecommunications Conference, vol. 2, San Francisco, CA, 27 September-1 December 2000, pp. 980984. 
[4] T. Eng and L. B. Milstein, "Coherent DS-CDMA Performance in Nakagami Multipath Fading," IEEE Transactions on Communications, vol. 43, no. 2/3/4, pp. 1134-1143, February/March/April 1995.

[5] E. Geraniotis and B. Ghaffari, "Performance of Binary and Quaternary Direct-Sequence Spread-Spectrum Multiple-Access Systems with Random Signature Sequences," IEEE Transactions on Communications, vol. 39, no. 5, pp. 713-724, May 1991.

[6] J. M. Holtzman, "A Simple, Accurate Method to Calculate SpreadSpectrum Multiple-Access Error Probabilities," IEEE Transactions on Communications, vol. 40, no. 3, pp. 461-464, March 1992.

[7] J. S. Lehnert and M. B. Pursley, "Error Probabilities for Binary DirectSequence Spread-Spectrum Communications with Random Signature Sequences," IEEE Transactions on Communications, vol. 35, no. 1, pp. 87-98, January 1987.

[8] _ , "Multipath Diversity Reception of Spread-Spectrum MultipleAccess Communications," IEEE Transactions on Communications, vol. 35, no. 11, pp. 1189-1198, November 1987.

[9] T. M. Lok and J. S. Lehnert, "Error Probabilities for Generalized Quadriphase DS/SSMA Communication Systems with Random Signature Sequences," IEEE Transactions on Communications, vol. 44, no. 7, pp. 876-885, July 1996.

[10] J. R. K. Morrow and J. S. Lehnert, "Bit-to-Bit Error Dependence in Slotted DS/SSMA Packet Systems with Random Signature Sequences," IEEE Transactions on Communications, vol. 37, no. 10, pp. 1052-1061, October 1989.

[11] K. Sivanesan and N. C. Beaulieu, "Performance Analysis of Bandlimited DS-CDMA Systems in Nakagami Fading," in IEEE International Conference on Communications, vol. 1, Paris, France, 20-24 June 2004, pp. $400-404$.

[12] M. O. Sunay and P. J. McLane, "Calculating Error Probabilities for DS-CDMA Systems: When Not to Use the Gaussian Approximation," in IEEE Global Telecommunications Conference, vol. 3, London, UK, 18-22 November 1996, pp. 1744-1749.

[13] — "Sensitivity of a DS CDMA System with Long PN Sequences to Synchronization Errors," in IEEE International Conference on Communications, vol. 2, Seattle, WA, USA, 18-22 June 1995, pp. 1029-1035.

[14] C. Unger and G. P. Fettweis, "Analysis of the RAKE Receiver Performance in Low Spreading Gain DS/SS Systems," in IEEE Global Telecommunications Conference 2002, vol. 1, 17-21 November 2002, pp. 830-834.

[15] Y. C. Yoon, "Quadriphase DS-CDMA with Pulse Shaping and the Accuracy of the Gaussian Approximation for Matched Filter Receiver Performance Analysis," IEEE Transactions on Wireless Communications, vol. 1, no. 4, pp. 761-768, October 2002

[16] A. Mirbagheri and Y. C. Yoon, "Performance Analysis of a Linear MMSE Receiver for Bandlimited Random-CDMA Using Quadriphase Spreading over Multipath Channels," IEEE Transactions on Wireless Communications, vol. 3, no. 4, pp. 1053-1066, July 2004.

[17] K. Sivanesan and N. C. Beaulieu, "Accurate BER Analysis of Bandlimited DS-CDMA System with EGC and SC Diversity over Nakagami Fading Channels," in IEEE Wireless Communications and Networking Conference, vol. 2, New Orleans, Louisiana, USA, 13-17 March 2005, pp. 956-960.

[18] E. A. Geraniotis and M. B. Pursley, "Error Probability for DirectSequence Spread-Spectrum Multiple-Access Communications-Part II: Approximations," IEEE Transactions on Communications, vol. 30, no. 5, pp. 985-995, May 1982.

[19] M. O. Sunay and P. J. McLane, "Comparison of Biphase Spreading to Quadriphase Spreading in DS CDMA Systems that Employ Long PN Sequences," in Sixth IEEE International Symposium on Personal, Indoor and Mobile Radio Communications, vol. 1, Toronto, Canada, 27-29 September 1995, pp. 237-242.

[20] — "Effects of Carrier Phase and Chip Timing Errors on the Capacity of a Quadriphase Spread BPSK Modulated DS-CDMA System," in IEEE Global Telecommunications Conference, vol. 2, Singapore, 13-17 November 1995, pp. 1114-1120.

[21] Q. Shi and M. Latva-Aho, "Accurate Bit-Error Rate Evaluation for Synchronous MC-CDMA over Nakagami- $m$-Fading Channels Using Moment Generating Functions," IEEE Transactions on Wireless Communications, vol. 4, no. 2, pp. 422-433, March 2005.

[22] F. D. Garber and M. B. Pursley, "Performance of Offset Quadriphase Spread-Spectrum Multiple-Access Communications," IEEE Transactions on Communications, vol. 29, no. 3, pp. 305-314, March 1981.

[23] R. T. Hsu and J. S. Lehnert, "A Characterization of Multiple-Access Interference in Generalized Quadriphase Spread-Spectrum Communications," IEEE Transactions on Communications, vol. 42, no. 2/3/4, pp. 2001-2010, FEBRUARY/MARCH/APRIL 1994.
[24] D. Laforgia, A. Luvison, and V. Zingarelli, "Bit Error Rate Evaluation for Spread-Spectrum Multiple-Access Systems," IEEE Transactions on Communications, vol. 32, no. 6, pp. 660-669, June 1984.

[25] M. B. Pursley, D. V. Sarwate, and W. E. Stark, "Error Probability for Direct-Sequence Spread-Spectrum Multiple-Access CommunicationsPart I: Upper and Lower Bounds," IEEE Transactions on Communications, vol. 30, no. 5, pp. 975-984, May 1982.

[26] M. B. Pursley, "Performance Evaluation for Phase-Coded SpreadSpectrum Multiple-Access Communication-Part I: System Analysis," IEEE Transactions on Communications, vol. 25, no. 8, pp. 795-799, August 1977.

[27] M. K. Simon and M.-S. Alouini, "A Unified Approach to the Performance Analysis of Digital Communication over Generalized Fading Channels," IEEE Proceedings, vol. 86, no. 9, pp. 1860-1877, September 1998.

[28] M. Nakagami, "The $m$-Distribution - A General Formula of Intensity Distribution of Rapid Fading," in Statistical Methods in Radio Wave Propagation, W. C. Hoffman, Ed. London: Pergamon Press, 1960, pp. 3-36.

[29] S. O. Rice, "Statistical Properties of a Sine Wave Plus Random Noise," Bell System Technical Journal, vol. 27, pp. 109-157, January 1948.

[30] A. Annamalai, C. Tellambura, and V. K. Bhargava, "Equal-Gain Diversity Receiver Performance in Wireless Channels," IEEE Transactions on Communications, vol. 48, no. 10, pp. 1732-1745, October 2000.

[31] A. Erdelyi, W. Magnus, F. Oberhettinger, and F. G. Tricomi, Eds., Higher Transcendental Functions. New York, Toronto and London: McGrawHill Book Company, Inc., 1953, vol. 1.

[32] H. Exton, Handbook of Hypergeometric Integrals - Theory, Applications, Tables, Computer Programs. Chichester, New York, Brisbane, Toronto: Ellis Horwood Ltd., 1978.

[33] H. M. Srivastava and P. W. Karlsson, Multiple Gaussian Hypergeometric Series. Ellis Horwood, Ltd., 1985.

[34] A. Papoulis, Probability, Random Variables, and Stochastic Processes, 3rd ed. McGraw-Hill, Inc., 1991.

[35] I. S. Gradshteyn and I. M. Ryzhik, Table of Integrals, Series, and Products, 6th ed., A. Jeffrey and D. Zwillinger, Eds. Academic Press, 2000

[36] K. Sivanesan and N. C. Beaulieu, "Exact BER Analysis of Bandlimited BPSK with EGC and SC Diversity in Cochannel Interference and Nakagami Fading," IEEE Communications Letters, vol. 8, no. 10, pp. 623-625, October 2004 